\title{
Retraction Note to: XBP1S protects cells from ER stress-induced apoptosis through Erk1/2 signaling pathway involving CHOP
}

\author{
Feng-Jin Guo $o^{1,2} \cdot Y_{a n n a ~ L i u^{1,2}}$ Jinghua Zhou ${ }^{1,2} \cdot$ Suxin Luo ${ }^{3} \cdot$ Wenjun Zhao ${ }^{1,2} \cdot$ Xiangzhu Li $^{1,2} \cdot$ Chuanju Liu $^{4}$
}

Published online: 1 July 2021

○) Springer-Verlag GmbH Germany, part of Springer Nature 2021

\section{Retraction to: Histochem Cell Biol (2012) 138:447-460 https://doi.org/10.1007/s00418-012-0967-7}

The Editor-in-Chief has retracted this article by Guo et al. (2012) because panels a, b and c of Fig. 1C appear to have been previously published (Guo et al. 2010) as Fig. 4A, XBP1U panel and XPB1S panel and Fig. 4B XPB1S panel respectively. Additionally, panel d of Fig. 5A appears to overlap with the $\beta$-actin panel of Fig. 1D in Li et al. (2012). The Editor-in-Chief has therefore concluded that the data reported in this article are unreliable.

Feng-Jin Guo disagrees with this retraction. The other authors have not responded to any correspondence regarding this retraction.

\section{References}

Guo Feng-Jin, Lin EA, Liu P, Lin J, Liu C (2010) XBP1U inhibits the XBP1S-mediated upregulation of the iNOS gene expression in mammalian ER stress response. Cell Signal 22(12):1818-1828. https://doi.org/10.1016/j.cellsig.2010.07.006

The original article can be found online at https://doi.org/10.1007/ s00418-012-0967-7.

Feng-Jin Guo

guo.fengjin@gmail.com

1 Department of Cell Biology and Genetics, Chongqing Medical University, Chongqing 400016, China

2 Core Facility of Development Biology, Chongqing Medical University, Chongqing 400016, China

3 Department of Cardiology, The First Affiliated Hospital, Chongqing Medical University, Chongqing 400016, China

4 Department of Orthopaedic Surgery and Department of Cell Biology, New York University School of Medicine, New York, NY 10016, USA
Li X, Zhu H, Huang H et al (2012) Study on the effect of IRE1 $\alpha$ on cell growth and apoptosis via modulation PLK1 in ER stress response. Mol Cell Biochem 365:99-108. https://doi.org/10.1007/ s11010-012-1248-4

Publisher's Note Springer Nature remains neutral with regard to jurisdictional claims in published maps and institutional affiliations. 